\title{
ecancermedicalscience \\ Are we ready for routine precision medicine? Highlights from the Milan Summit on Precision Medicine, Milan, Italy, 8-9 February 2018
}

\author{
Luca Mazzarella \\ European Institute of Oncology, Via Ripamonti 435, Milan 20141, Italy \\ Correspondence to: Luca Mazzarella. Email: luca.mazzarella@ieo.eu
}

\begin{abstract}
On 8 and 9 February 2018, the IFOM-IEO campus in Milan hosted the Milan summit on Precision Medicine, which gathered clinical and translational research experts from academia, industry and regulatory bodies to discuss the state of the art of precision medicine in Europe. The meeting was pervaded by a generalised feeling of excitement for a field that is perceived to be technologically mature for the transition into clinical routine but still hampered by numerous obstacles of a methodological, ethical, regulatory and possibly cultural nature. Through lively discussions, the attendees tried to identify realistic ways to implement a technology-rich precision approach to cancer patients.
\end{abstract}

Keywords: genomics, next generation sequencing, NGS, precision medicine, targeted therapy

Published: 05/03/2018

Received: 19/02/2018

ecancer 2018, 12:817 https://doi.org/10.3332/ecancer.2018.817

Copyright: (c) the authors; licensee ecancermedicalscience. This is an Open Access article distributed under the terms of the Creative Commons Attribution License (http://creativecommons.org/licenses/by/3.0), which permits unrestricted use, distribution, and reproduction in any medium, provided the original work is properly cited. 


\section{Introduction}

The concept of 'precision medicine', as highlighted by Professor De Braud (Istituto Nazionale dei Tumori (INT), Italy) at the summit, has transitioned in recent years from an initial focus on tumour genetic profiling and targeted drugs to a broader definition encompassing multiple phases of cancer natural history, including prevention, early diagnosis, therapy and surveillance. Although several studies have shown that a precision medicine approach can significantly improve patient outcomes, widespread adoption is made difficult by the complex, multidisciplinary nature of the required expertise and the need for adequate support of a financial, organisational and administrative nature. The summit brought together experts with heterogeneous backgrounds to discuss how to overcome current barriers. Many speakers provided an overview of ongoing large-scale multi-institutional projects assessing the feasibility and clinical benefit of next-generation sequencing (NGS) and other high-throughput molecular analytical approaches. An overview of recent technological improvement was provided, especially on the growing sophistication and utility of liquid biopsies. Finally, some speakers concentrated on novel biological understanding obtained from molecular characterisation.

\section{Multi-institutional precision medicine projects}

Large-scale sequencing projects are being conducted in several countries [1]. Professor PG Pelicci (European Institute of Oncology (IEO), Italy) reviewed the experience of the Alleanza Contro il Cancro (ACC) consortium in Italy, which groups together 21 cancer centres. ACC recently launched a nationwide pilot trial in lung cancer that aims at collecting and analysing detailed genetic information from 1,000 patients, using a custom-designed targeted sequencing panel focused on actionable mutations. Beside evaluating the feasibility and accuracy of large-scale sequencing in a disease in which genetic stratification is a key to adequate treatment, the programme will systematically collect samples for longitudinal studies on biomarkers of treatment response. The consortium will then apply an evolution of this approach to multiple other diseases, collecting all clinical and medical information in a national repository that will allow the development of a computational platform to assist in clinical decision making and will accelerate patient enrolment to clinical trials. Similar efforts are ongoing in Spain, as highlighted by Dr Garrido (Universidad de Alcalá, Madrid, Spain) [2].

Dr Tonu Esko (Estonian Genome Center, Estonia) provided an overview of the Estonian program for personalised medicine, which aims to obtain detailed molecular profiling of 52,000 adults (5\% of the adult population) followed longitudinally with direct matching to electronic medical records. Thousands of these subjects are expected to be characterised by whole genome/whole exome sequencing, transcriptomics and metabolomics. Although the ethical implications of matching genetic information with medical information are evident, a survey showed that an overwhelming majority (>70\%) of interviewees support the project, suggesting that ethical concerns can be overcome if the expected return is tangible.

Dr Kramer highlighted the role that molecular profiling based on gene expression [3, 4] or DNA methylation [5] can have in identifying the tissue of origin and best treatment in cases of cancer of unknown primary, a heterogeneous group of diseases for which the current median overall survival is in the order of a few months [6]. NGS of tumour DNA [7] and analysis of cfDNA [8] can identify actionable mutations and elevated tumour mutational burden [9] in $\sim 10-30 \%$ of the cases, offering a valid therapeutic alternative to chemotherapy as demonstrated by several case reports [10,11]. Strategies to personalise treatment based on gene expression [9] or NGS (MX39795 trial) are ongoing.

Prof Andrew Hughes from the Christie Hospital (Manchester, UK) provided an overview of the TARGET trial in the UK, aimed at validating liquid biopsy for metastatic disease monitoring and treatment allocation. He highlighted six groups of issues faced by precision medicine programmes: ethical, administrative, technological, scientific, financial and organisational. Besides scientific and technological issues related to reliability of the assay and interpretation of the result, discussed by many others, he identified other crucial aspects worth mentioning. First, the crucial ethical issue of managing information returns to the patient, which TARGET decided to deliver using the 'flexible-default' model $[12,13]$. Second, the availability of electronic databases and medical records, which are key to allowing information sharing and decision making within the Tumour Molecular Board. Finally, the importance of activating a fair number of biomarker-driven clinical trials to allocate patients based on the molecular profile: increase in trial availability resulted in an increased allocation rate from $0 \%$ in 2012 to $18 \%$ in 2016. This translates into a real clinical benefit for patients, as meta-analyses of phase 1, 2 and 3 trials demonstrate an improvement in outcome measures when patients are allocated to biomarker-driven trials, as opposed to nonbiomarker-driven [14-16]. A sobering point of view was provided by Dr Le Tourneau (Institut Curie Paris, France) who pointed out that much of the benefit observed in these meta-analyses is actually limited to FDA-approved drugs and is less clear for experimental drugs at earlier phases of development. 
Still, many attendees expressed the view that current access to targeted therapy is inadequate and, in light of the demonstrated clinical benefit, increased reimbursement for high-throughput molecular testing is justified.

Talks by Professor De Braud, Dr Biganzoli (University of Milan, Italy) and Professor Curigliano (European Institute of Oncology (IEO), Italy) dwelled on the changing face of drug development in the framework of personalised medicine, with an increased usage of adaptive trial designs that allow testing of multiple hypotheses at once, so as to maximise costs and patient enrolment [17]. The speakers called for a new comprehensive strategy that overcomes the current single drug-driven model, a strategy best promoted by academia, regulatory bodies and the government in collaboration with pharmaceutical companies.

Two talks were held by representatives of the European (EMA) and Italian (AIFA) regulatory bodies. A hotly discussed topic was that of histology-agnostic biomarkers. Even if considered very biologically relevant, many are insufficiently supported by clinical trials to be clearly recommended into clinical practice. Prominent examples are programmed death ligand 1 (PDL1) expression, troubled by inter-assay technical variability and a heterogeneous predictive power in different diseases [18] and BRCA1/2 mutational status, insufficient to identify responders to poly-ADP ribose polymerase inhibitors [19].

An intriguing talk by the psychologist Ketti Mazzocco (University of Milan, Italy) highlighted the importance of 'framing' information to patients and how appropriate communication strategies can increase the chances of a patient's acceptance of precision medicine approaches.

\section{Technological advancements}

Drs Ross and Pavlick from Foundation Medicine, the private company that pioneered implementation of NGS for precision oncology, shared their experience on thousands of patients analysed through their commercially available platforms. Their NGS-based assay led to the identification of several genetic alterations actionable for targeted treatment [20-22]. They identified many technical barriers to precision medicine: the inter- and intra-individual genomic complexity, requiring adequately sized sequencing panels; the difficulty in obtaining adequate bioptic material; assay sensitivity, crucial since false negatives will cause patients to be treated with significantly more toxic and/ or less effective therapy. Discordance rate between NGS-based and conventional, approved non-NGS analytical techniques is extremely high, up to $35 \%$ for anaplastic lymphoma kinase translocations.

A wide overview on liquid biopsy was presented by Dr Klaus Pantel (University Medical Centre Hamburg-Eppendorf, Germany), a pioneer in the field. Liquid biopsy is an umbrella name that encompasses different assays detecting circulating tumour cells (CTC), tumour DNA (ctDNA) or other biologically relevant moleculaes (microRNAs, exosomes, metabolites, etc.). Liquid biopsies can provide useful information in various oncological settings: early detection and screening, prognostication by quantifying risk of relapse/progression, real-time monitoring of therapies and identification of resistance mechanisms [23, 24]. Perhaps a poorly recognised issue for early detection/screening is the high rate of background positivity for some common mutations such as TP53 [25, 26], probably reflecting precancerous lesions in apparently healthy subjects. A combination of multiple markers is likely to provide increased specificity [27]. In the setting of tumour prognostication, CTCs are certainly the most developed technology $[28,29]$, to the point that some authors advocate implementing CTC counts in the TNM staging system. Additional useful biological information can be retrieved from CTCs, to be used to drive therapy with immune checkpoint inhibitors (like PDL1 expression) or to better decide on endocrine therapy in metastatic breast cancer, since CTC often show heterogeneous estrogen receptor status [30,31]. CtDNA analysis has become established in specific settings, like the identification of T790M resistanceinducing mutations in epidermal growth factor receptor (EGFR)-mutated lung cancer. However, the currently approved methodology remains poorly sensitive, with up to $30 \%$ false-negatives [32]. But as pointed out by Jeff Ross from Foundation Medicine, the information retrievable from cfDNA-based liquid biopsies goes beyond individual mutational hotspots, including for instance Tumour Mutational Burden that can identify responders to immune checkpoint inhibitors [33].

\section{Biological advancements}

Professor Foiani from the IFOM institute in Milan discussed newly discovered roles of ataxia telangiectasia and Rad3-related protein/ataxia telangiectasia mutated (ATR/ATM) kinases, well known to be involved in maintenance of DNA stability and recently found by himself and colleagues to play a role in tumour migration and metastasis [34]. 
Dr Bedognetti (Sidra Medicine, Doha, Qatar) reviewed the progress brought about by NGS in the understanding of tumour microenvironment and immunity. RNA expression studies identified gene modules over-represented in various immune phenomena (like autoimmunity or rejection) and associated with different outcomes and/or response to immunotherapy in multiple tumours, which are referred to as immunological constant of rejection (ICR). By analysing these modules in breast cancer, they identified 4 ICR subgroups (ICR1-4) that variably correlated with genomic features associated with differential response to immunotherapy (mutational burden, specific mutations in Molecular Analysis for Personalized Medicine kinases, structural aberrations) [35]. These gene expression-based profiles are evolving into clinical-grade assays to be used for predicting sensitivity to immunotherapy [36], especially in poorly immunogenic diseases like breast cancer. This and similar studies have led to novel therapeutic combinations that may turn immunologically 'cold', nonreponsive tumours into 'hot', immune therapy-sensitive ones [37]

\section{Funding}

The author was remunerated for writing this conference report through an unrestricted educational grant provided by Roche Foundation Medicine.

\section{References}

1. ICPerMed International Consortium (2017) ICPerMed action plan for personalised medicine [http://www.icpermed.eu/media/content/ICPerMed Actionplan 2017 web.pdf] Date accessed: 15/02/2018

2 Garrido P, Aldaz A, and Calleja MÁ, et al (2017) Proposal for the creation of a national strategy for precision medicine in cancer: a position statement of SEOM, SEAP and SEFH Farm Hosp 41 688-691

3. Hainsworth JD, Rubin MS, and Spigel DR, et al (2013) Molecular gene expression profiling to predict the tissue of origin and direct site-specific therapy in patients with carcinoma of unknown primary site: a prospective trial of the Sarah Cannon Research Institute J Clin Oncol 31 217-223 https://doi.org/10.1200/JCO.2012.43.3755

4. Yoon HH, Foster NR, and Meyers JP, et al (2016) Gene expression profiling identifies responsive patients with cancer of unknown primary treated with carboplatin, paclitaxel, and everolimus: NCCTG N0871 (alliance) Ann Oncol 27 339-344 https:// doi.org/10.1093/annonc/mdv543 PMCID: 4907341

5. Moran S, Martínez-Cardús A, and Sayols S, et al (2016) Epigenetic profiling to classify cancer of unknown primary: a multicentre, retrospective analysis Lancet Oncol 17 1386-1395 https://doi.org/10.1016/S1470-2045(16)30297-2 PMID: 27575023

6. Riihimäki M, Hemminki A, and Sundquist K, et al (2013) Time trends in survival from cancer of unknown primary: small steps forward Eur J Cancer 49 2403-2410 https://doi.org/10.1016/j.ejca.2013.02.022 PMID: 23518210

7. Varghese AM, Arora A, and Capanu M, et al (2017) Clinical and molecular characterization of patients with cancer of unknown primary in the modern era Ann Oncol 28 3015-3021 https://doi.org/10.1093/annonc/mdx545 PMID: 29045506

8. Kato S, Krishnamurthy N, and Banks KC, et al (2017) Utility of genomic analysis in circulating tumor DNA from patients with carcinoma of unknown primary Cancer Res 77(16) 4238-4246 https://doi.org/10.1158/0008-5472.CAN-17-0628 PMID: 28642281 PMCID: $\underline{5729906}$

9. Gay LM, Fabrizio D, and Frampton GM, et al (2017) Mutational burden of tumors with primary site unknown J Clin Oncol 353039 https://doi.org/10.1200/JCO.2017.35.15 suppl.3039

10. Rose JS, Wang K, and Gay L, et al (2015) Comprehensive genomic profiling of carcinoma of unknown primary site: new routes to targeted therapies JAMA Oncol 1 40-49 https://doi.org/10.1001/jamaoncol.2014.216 
11. Palma NA, Ali SM, and O'Connor J, et al (2014) Durable response to crizotinib in a MET-amplified, KRAS-mutated carcinoma of unknown primary Case Rep Oncol 7 503-508 https://doi.org/10.1159/000365326 PMID: 25232318 PMCID: 4164090

12. Dressler LG (2009) Disclosure of research results from cancer genomic studies: state of the science Clin Cancer Res 15 4270-4276 https://doi.org/10.1158/1078-0432.CCR-08-3067 PMID: 19549775

13. Halpern SD, Ubel PA, and Asch DA (2007) Harnessing the power of default options to improve health care N Engl J Med 357 1340-1344 https://doi.org/10.1056/NEJMsb071595 PMID: 17898105

14. Schwaederle M, Zhao M, and Lee JJ, et al (2016) Association of biomarker-based treatment strategies with response rates and progression-free survival in refractory malignant neoplasms: a meta-analysis JAMA Onco/ 2 1452-1459 https://doi.org/10.1001/ jamaoncol.2016.2129 PMID: 27273579

15. Schwaederle M, Zhao M, and Lee JJ, et al (2015) Impact of precision medicine in diverse cancers: a meta-analysis of phase II clinical trials J Clin Oncol 33 3817-3825 https://doi.org/10.1200/JC0.2015.61.5997 PMID: 26304871 PMCID: 4737863

16. Jardim DL, Schwaederle M, and Wei C, et al (2015) Impact of a biomarker-based strategy on oncology drug development: a meta-analysis of clinical trials leading to FDA approval J Natl Cancer Inst 107(11) PMID: 26378224 PMCID: 4857149

17. Freidlin B, McShane LM, and Korn EL (2010) Randomized clinical trials with biomarkers: design issues $J$ Natl Cancer Inst 102 152-160 https://doi.org/10.1093/jnci/djp477 PMID: 20075367 PMCID: 2911042

18. Patel SP and Kurzrock R (2015) PD-L1 expression as a predictive biomarker in cancer immunotherapy Mol Cancer Ther 14 847-856 https://doi.org/10.1158/1535-7163.MCT-14-0983 PMID: 25695955

19. Mirza MR, Monk BJ, and Herrstedt J, et al (2016) Niraparib maintenance therapy in platinum-sensitive, recurrent ovarian cancer N Engl J Med [doi:10.1056/NEJMoa1611310] https://doi.org/10.1056/NEJMoa1611310 PMID: 27717299

20. Drilon A, Wang L, and Arcila ME, et al (2015) Broad, hybrid capture-based next-generation sequencing identifies actionable genomic alterations in lung adenocarcinomas otherwise negative for such alterations by other genomic testing approaches Clin Cancer Res 21 3631-3639 https://doi.org/10.1158/1078-0432.CCR-14-2683 PMID: 25567908 PMCID: 4917003

21. Frampton GM, Ali SM, and Rosenzweig M, et al (2015) Activation of MET via diverse exon 14 splicing alterations occurs in multiple tumor types and confers clinical sensitivity to MET inhibitors Cancer Discov 5 850-859 https://doi.org/10.1158/2159-8290. CD-15-0285 PMID: 25971938

22. Drilon A, Wang L, and Hasanovic A, et al (2013) Response to cabozantinib in patients with RET fusion-positive lung adenocarcinomas Cancer Discov 3 630-635 https://doi.org/10.1158/2159-8290.CD-13-0035 PMID: 23533264 PMCID: 4160032

23. Bardelli A and Pantel K (2018) Liquid biopsies, what we do not know (yet) Cancer Cell 31 172-179 https://doi.org/10.1016/j. ccell.2017.01.002

24. Alix-Panabieres $C$ and Pantel K (2016) Clinical applications of circulating tumor cells and circulating tumor DNA as liquid biopsy Cancer Discov 6 479-491 https://doi.org/10.1158/2159-8290.CD-15-1483 PMID: 26969689

25. Krimmel JD, Schmitt MW, and Harrell MI, et al (2016) Ultra-deep sequencing detects ovarian cancer cells in peritoneal fluid and reveals somatic TP53 mutations in noncancerous tissues Proc Natl Acad Sci 113 6005-6010 https://doi.org/10.1073/ pnas.1601311113 PMID: 27152024 PMCID: 4889384

26. Fernandez-Cuesta L, Perdomo S, and Avogbe $\mathrm{PH}$, et al (2016) Identification of circulating tumor DNA for the early detection of small-cell lung cancer EBioMedicine 10 117-123 https://doi.org/10.1016/j.ebiom.2016.06.032 PMID: 27377626 PMCID: 5036515

27. Cohen JD, Li L, and Wang Y, et al (2018) Detection and localization of surgically resectable cancers with a multi-analyte blood test Science https://doi.org/10.1126/science.aar3247 
28. Lorente D, Olmos D, and Mateo J, et al (2016) Decline in circulating tumor cell count and treatment outcome in advanced prostate cancer Eur Urol 70 985-992 https://doi.org/10.1016/j.eururo.2016.05.023 PMID: 27289566 PMCID: 5568108

29. Bidard FC, Peeters DJ, and Fehm T, et al (2014) Clinical validity of circulating tumour cells in patients with metastatic breast cancer: a pooled analysis of individual patient data Lancet Oncol 15 406-414 https://doi.org/10.1016/S1470-2045(14)70069-5 PMID: 24636208

30. Babayan A, Hannemann J, and Spötter J, et al (2013) Heterogeneity of estrogen receptor expression in circulating tumor cells from metastatic breast cancer patients PLoS One 8 e75038 https://doi.org/10.1371/journal.pone.0075038 PMID: 24058649 PMCID: $\underline{3776726}$

31. Mazel M, Jacot W, and Pantel K, et al (2015) Frequent expression of PD-L1 on circulating breast cancer cells Mol Oncol 9 1773-1782 https://doi.org/10.1016/j.molonc.2015.05.009 PMID: 26093818 PMCID: 5528721

32. Oxnard GR, Thress KS, and Alden RS, et al (2016) Association between plasma genotyping and outcomes of treatment with osimertinib (AZD9291) in advanced non-small-cell lung cancer J Clin Oncol 34 3375-3382 https://doi.org/10.1200/

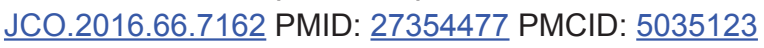

33. Khagi Y, Goodman AM, and Daniels GA, et al (2017) Hypermutated circulating tumor DNA: correlation with response to checkpoint inhibitor-based immunotherapy Clin Cancer Res 23 5729-5736 https://doi.org/10.1158/1078-0432.CCR-17-1439 PMID: $\underline{28972084}$ PMCID: $\underline{5678984}$

34. Awasthi P, Foiani M, and Kumar A (2015) ATM and ATR signaling at a glance J Cell Sci 128 4255-4262 https://doi.org/10.1242/ ics. 169730 PMID: 26567218

35. Hendrickx W, Simeone I, and Anjum S, et al (2017) Identification of genetic determinants of breast cancer immune phenotypes by integrative genome-scale analysis Oncoimmunology 6 e1253654 https://doi.org/10.1080/2162402X.2016.1253654 PMID: $\underline{28344865}$ PMCID: $\underline{5353940}$

36. Ayers M, Lunceford J, and Nebozhyn M, et al (2017) IFN- $y$-related mRNA profile predicts clinical response to PD-1 blockade $J$ Clin Invest 127 2930-2940 https://doi.org/10.1172/JCI91190 PMID: 28650338 PMCID: 5531419

37. Dushyanthen S, Teo ZL, and Caramia F, et al (2017) Agonist immunotherapy restores T cell function following MEK inhibition improving efficacy in breast cancer Nat Commun 8606 https://doi.org/10.1038/s41467-017-00728-9 PMID: 28928458 PMCID: $\underline{5605577}$ 